\title{
Infinite Paths of Minimal Length on Suborbital Graphs for Some Fuchsian Groups
}

\author{
Khuanchanok Chaichana ${ }^{1}$ and Pradthana Jaipong $\mathbb{D}^{2}$ \\ ${ }^{1}$ Degree Program in Mathematics, Faculty of Science, Chiang Mai University, Chiang Mai 50200, Thailand \\ ${ }^{2}$ Center of Excellence in Mathematics and Applied Mathematics, Department of Mathematics, Faculty of Science, \\ Chiang Mai University, Chiang Mai 50200, Thailand
}

Correspondence should be addressed to Pradthana Jaipong; pradthana.j@cmu.ac.th

Received 21 December 2018; Revised 8 February 2019; Accepted 23 August 2019; Published 3 October 2019

Academic Editor: Niansheng Tang

Copyright ( 2019 Khuanchanok Chaichana and Pradthana Jaipong. This is an open access article distributed under the Creative Commons Attribution License, which permits unrestricted use, distribution, and reproduction in any medium, provided the original work is properly cited.

In this study, we work on the Fuchsian group $H \sqrt{m}$ where $m$ is a prime number acting on $\sqrt{m} \widehat{\mathbb{Q}}$ transitively. We give necessary and sufficient conditions for two vertices to be adjacent in suborbital graphs induced by these groups. Moreover, we investigate infinite paths of minimal length in graphs and give the recursive representation of continued fraction of such vertex.

\section{Introduction}

The Hecke group, $H(\lambda)$, introduced by Hecke in [1], is the group generated by the two Möbius transformations

$$
\begin{aligned}
& R(z)=-\frac{1}{z}, \\
& S(z)=z+\lambda,
\end{aligned}
$$

where $\lambda$ is a real number such that $\lambda=\lambda_{q}=2 \cos (\pi / q)$ and $q$ is an integer greater than 2 or $\lambda>2$. When $q=3, H\left(\lambda_{3}\right)=$ $H(1)$ is the modular group $\operatorname{PSL}(2, \mathbb{Z})$. If $q=4,6$, it is known, see [2], that $H\left(\lambda_{q}\right)=H(\sqrt{m})$, where $m=2,3$ consists of all transformations of the following two types:

$$
\begin{gathered}
T(z)=\frac{a z+b \sqrt{m}}{c \sqrt{m} z+d}, \quad a, b, c, d \in \mathbb{Z}, a d-b c m=1, \\
T(z)=\frac{a \sqrt{m} z+b}{c z+d \sqrt{m}}, \quad a, b, c, d \in \mathbb{Z}, a d m-b c=1 .
\end{gathered}
$$

However, Rosen [3] showed that the above two transformations need not to be in $H(\lambda)$ if $\lambda \neq 1, \sqrt{2}, \sqrt{3}$. He proved that $T$ is an element of $H(\lambda)$ if and only if $a / c$ is a finite $\lambda$-fraction, i.e.,

$$
\frac{a}{c}=r_{0} \lambda-\frac{1}{r_{1} \lambda-\frac{1}{r_{2} \lambda-\frac{1}{\ddots \quad-\frac{1}{r_{\mathrm{n}} \lambda}}}}
$$

where $r_{0}$ is an integer and $r_{i}$ is a positive integer for $i=1, \ldots, n$. Later, Keskin [4] presented the Fuchsian group $H(\sqrt{m})$ for a squarefree positive integer $m$, which consists of all mappings of the forms (2) and (3).

In 1991, Jones et al. [5] studied the modular group $H(1)$ by applying the idea of the suborbital graphs for a permutation group, introduced in [6]. Later, Akbas [7] proved his conjecture stating that a suborbital graph for the modular group is a forest if and only if it contains no triangles. They completely characterized circuits in suborbital graphs for the modular group. These studies lead us to explore infinite paths in suborbital graphs for the Fuchsian group $H(\sqrt{m})$.

In [8], Yayenie gave a remark stating that the Fuchsian group $H(\sqrt{m})$ acts transitively on the set $\sqrt{m} \widehat{\mathbb{Q}}$ if and only if 
$m$ is either 1 or prime. So, we study on the Fuchsian group $H(\sqrt{m})$ where $m$ is a prime number. We divide this work into four sections. In Section 2, we will show the way to construct a suborbital graph $G(\infty,(u / v) \sqrt{m})$ for the group $H(\sqrt{m})$ and we give the edge conditions for two vertices that are joined in the graph. In Sections 3 and 4, we investigate vertices on the infinite path of minimal length in the graph $G(\infty,(u / v) \sqrt{m})$ for two cases: $(m, v)=1$ and $(m, v)=m$, respectively; here, ordered pairs denote greatest common divisors. Finally, we represent each vertex on the infinite path of minimal length by recurrence relations and determine the limit point of the sequence of the vertices.

\section{Suborbital Graphs for $H(\sqrt{m})$}

Let $\widehat{\mathbb{Q}}=\mathbb{Q} \cup\{\infty\}$ and $m$ be a squarefree positive integer, every element of $\sqrt{m} \widehat{\mathbb{Q}}$ can be represented as a fraction $(x / y) \sqrt{m}$ with $x, y \in \mathbb{Z}$ and $(x, y)=1$. We represent $\infty$ as $(1 / 0) \sqrt{m}=(-1 / 0) \sqrt{m} . H(\sqrt{m})$ acts on $\sqrt{m} \widehat{\mathbb{Q}}$ naturally by

$$
T \cdot \frac{x}{y} \sqrt{m}=T\left(\frac{x}{y} \sqrt{m}\right),
$$

$T \in H(\sqrt{m}),(x / y) \sqrt{m} \in \sqrt{m} \widehat{\mathbb{Q}}$. The following remark was given by Yayanie in [8], regarding the Fuchsian group $H(\sqrt{m})$ acting transitively on vertices $\sqrt{m} \widehat{\mathbb{Q}}$.

Remark 1 [8]. The Fuchsian group $H(\sqrt{m})$ acts transitively on the set $\sqrt{m} \widehat{\mathbb{Q}}$ if and only if $m$ is either 1 or prime.

From now on, it will be assumed that $m$ is prime. So, $H(\sqrt{m})$ acts transitively on $\sqrt{m} \widehat{\mathbb{Q}}$. We will give a construction of suborbital graphs for the Fuchsian group $H(\sqrt{m})$. Let $H(\sqrt{m})$ act on $\sqrt{m} \widehat{\mathbb{Q}} \times \sqrt{m} \widehat{\mathbb{Q}}$ by

$$
g(\alpha, \beta)=(g(\alpha), g(\beta)) \text {. }
$$

$g \in H(\sqrt{m}), \alpha, \beta \in \sqrt{m} \widehat{\mathbb{Q}}$. The orbits of this action are called suborbitals of $H(\sqrt{m})$. The suborbital containing $(\alpha, \beta)$ is denoted by $O(\alpha, \beta)$. We can form a suborbital graph $G(\alpha, \beta)$ whose vertices are the elements of $\sqrt{m} \widehat{\mathbb{Q}}$, and there is a directed edge from $\lambda$ to $\delta$ if $(\gamma, \delta) \in O(\alpha, \beta)$, denoted by $\gamma \longrightarrow \delta$. We can see that $O(\beta, \alpha)$ is also a suborbital such that $O(\beta, \alpha)=O(\alpha, \beta)$ or $O(\beta, \alpha) \cap O(\alpha, \beta)=\varnothing$. In the latter case, $G(\beta, \alpha)$ is just $G(\alpha, \beta)$ with reversed arrows and we call $G(\beta, \alpha)$ and $G(\alpha, \beta)$ paired suborbital graphs. In the case $G(\beta, \alpha)=G(\alpha, \beta)$, the graph consists of pairs of oppositely directed edges, and we replace each pair with an undirected edge for convenience. We call the graph self-paired.

Since $H(\sqrt{m})$ acts on $\sqrt{m} \widehat{\mathbb{Q}}$ transitively, each suborbital contains a pair $(\infty,(u / v) \sqrt{m})$ for some $(u / v) \sqrt{m} \in \sqrt{m} \widehat{\mathbb{Q}}$. The following two theorems are valid for prime number $m$ and we can use the same technique in the proofs of Theorem 1 and 2 in [9], which were stated for $m=2$ and 3 .

Theorem 1. Let $u$ and $v$ be relatively prime and $m$ prime. If $(m, v)=1$, then, there exists a directed edge from $(r / s) \sqrt{m}$ to $(x / y) \sqrt{m}$ in $G(\infty,(u / v) \sqrt{m})$ if and only if $r y-s x= \pm v$ and either.

(i) $x \equiv \pm$ ur $\bmod v, y \equiv \pm$ us $\bmod v$ and $m \mid s$ or

(ii) $x \equiv \pm m u r \bmod v, y \equiv \pm m u s \bmod v$ and $m \mid y$.
Theorem 2. Let $u$ and $v$ be relatively prime and $m$ prime. If $(m, v)=m$, then, there exists a directed edge from $(r / s) \sqrt{m}$ to $(x / y) \sqrt{m}$ in $G(\infty,(u / v) \sqrt{m})$ if and only if either.

(i) $r y-s x= \pm v, m \mid s$ and $x \equiv \pm u r \bmod v, y \equiv \pm$ us $\bmod v$ or

(ii) $r y-s x= \pm(v / m)$ and $x \equiv \pm u r \bmod (v / m), y \equiv \pm$ us $\bmod v$.

Here, the choice of signs for $x$ and $y$ are always the same. By using Theorems 1 and 2, we obtain the following two corollaries that characterize a self-paired graph.

Corollary 1. Let $u$ and $v$ be relatively prime and $m$ prime such that $(m, v)=1$. Then the suborbital graph $G(\infty$, $(u / v) \sqrt{m})$ is self-paired if and only if $m u^{2}+1 \equiv 0 \bmod v$.

Corollary 2. Let $u$ and $v$ be relatively prime and $m$ prime such that $(m, v)=m$. Then the suborbital graph $G(\infty,(u / v)$ $\sqrt{m})$ is self-paired if and only if $u^{2}+1 \equiv 0 \bmod v$.

Next, we will show the existence of an integer $k$ such that $m u^{2}+k m u+1 \equiv 0 \bmod v$.

Lemma 1. Let $u$ and $v$ be relatively prime and $m$ prime such that $(m, v)=1$. Then there exist integers $k$ and $l$ with $1<k$, $l \leq v$ such that $m u^{2}+k m u+1 \equiv 0 \bmod v$ and $m u^{2}-l m u+1$ $\equiv 0 \bmod v$.

Proof. Since $(m, v)=(u, v)=1$, we have $(m u, v)=1$. Then, there exists an integer $x$ such that $\operatorname{mux} \equiv 1 \bmod v$. So $\operatorname{mux}\left(-m u^{2}-1\right) \equiv-m u^{2}-1 \bmod v$. Taking $k=x\left(-m u^{2}-\right.$ $1)$, it is seen that $m u^{2}+k m u+1 \equiv 0 \bmod v$ is satisfied. Note that $k$ and $l$ are uniquely determined.

Theorem 3. Let $u$ and $v$ be relatively prime and $m$ prime such that $(m, v)=1$. Suppose that $m u^{2}+k m u+1 \equiv 0 \bmod v$ and $m u^{2}-l m u+1 \equiv 0 \bmod v$ where $k, l$ are integers such that $1<k, l \leq v$. If $G(\infty,(u / v) \sqrt{m})$ is self-paired, then $k=l=v$; otherwise, $l=v-k$.

Proof. Since $m u^{2}+k m u+1 \equiv 0 \bmod v$ and $m u^{2}-l m u+$ $1 \equiv 0 \bmod v, k m u \equiv-l m u \bmod v$. As $(u, v)=1=(m, v)$, we obtain that $k \equiv-l \bmod v$. So, there exists an integer $y$ such that $k+l=v y$ and then $2<v y \leq 2 v$ since $1<k, l \leq v$. Hence, $y=1$ or 2 . Assume that $G(\infty,(u / v) \sqrt{m})$ is self-paired. By Corollary 1 , we have $m u^{2}+1 \equiv 0 \bmod v$ which implies that $k u \equiv 0 \bmod v$ and $-l u \equiv 0 \bmod v$. Since $v \nmid u, v \mid k$, and $v \mid l$. From $1<k, l \leq v$, we get $k=v=l$, so $y=2$. For the case $y=1$, we have $l=v-k$.

Lemma 2. Let $u$ and $v$ be relatively prime and $m$ prime. Then there exist integers $k$ and $l$ with $1<k, l \leq v$ such that $u^{2}+$ $k u+1 \equiv 0 \bmod v$ and $u^{2}-l u+1 \equiv 0 \bmod v$.

Theorem 4. Let $u$ and $v$ be relatively prime and $m$ prime. Suppose that $u^{2}+k u+1 \equiv 0 \bmod v$ and $u^{2}-l u+1 \equiv 0$ $\bmod v$ where $k, l$ are integers such that $1<k, l \leq v$. If $G(\infty$, $(u / v) \sqrt{m})$ is self-paired, then $k=l=v$; otherwise, $l=v-k$. 


\section{Infinite Path of Minimal Length in $G(\infty,(u / v) \sqrt{m})$ Where $(m, v)=1$}

Let $v_{1}, v_{2}, v_{3}, \ldots, v_{n}$ be vertices of the suborbital graph $G(\infty,(u / v) \sqrt{m})$, we call the configurations

$$
\begin{aligned}
& v_{1} \longrightarrow v_{2} \longrightarrow \cdots \longrightarrow v_{n}, \\
& v_{1} \longrightarrow v_{2} \longrightarrow v_{3} \longrightarrow \cdots,
\end{aligned}
$$

a path and an infinite path, respectively. If $v_{i} \stackrel{>}{\longrightarrow}$ (or $v_{i} \ll v_{j}$ ) and there is no vertex which has greater (or smaller) value than $v_{j}$ joined with the vertex $v_{i}$, then $v_{j}$ is the farthest vertex which can be joined with the vertex $v_{i}$. The path $v_{1} \longrightarrow v_{2} \longrightarrow \cdots \longrightarrow v_{n}$ is called of minimal length if and only if $v_{i} \leftrightarrow v_{j}$, where $i<j-1, i \in\{1,2,3, \ldots, n-2\}$, $j \in\{3,4,5, \ldots, n\}$ and $v_{i+1}$ must be the farthest vertex which can be joined with the vertex $v_{i}$.

In this section, we focus on the infinite path of minimal length in the suborbital graph $G(\infty,(u / v) \sqrt{m})$ where $(m, v)=1$. By the choice of prime, $m$, and remark 1 , we obtain transitivity. Thus, we can map the first edge of any infinite path to the edge $\infty \longrightarrow(u / v) \sqrt{m}$. We start investigating vertices in the infinite path of minimal length by determining the farthest vertex which can be joined with the vertex $(u / v) \sqrt{m}$.

Theorem 5. Let $u$ and $v$ be relatively prime and $m$ prime such that $(m, v)=1$, and let $k, l$ be the integers uniquely determined in Theorem 6 . Then, we have the following results in $G(\infty,(u / v) \sqrt{m})$ :

(i) The farthest vertices which can be joined with $(u / v) \sqrt{m}$ on the right and the left are

$$
\begin{aligned}
& \frac{u+(1 / k m)}{v} \sqrt{m}, \\
& \frac{u-(1 / l m)}{v} \sqrt{m},
\end{aligned}
$$

respectively. No nearest vertex exists.

(ii) The farthest vertices which can be joined with $((u+$ $(1 / \mathrm{km})) / v) \sqrt{m}$ and $((u-(1 / \mathrm{lm})) / v) \sqrt{m}$ are

$$
\begin{aligned}
& \frac{u+(1 /(k m-(1 / k)))}{v} \sqrt{m} \\
& \frac{u-(1 /(l m-(1 / l)))}{v} \sqrt{m}
\end{aligned}
$$

respectively. No nearest vertex exists.

(iii) The farthest vertices which can be joined with $((u+$ $(1 /(k m-(1 / k)))) / v) \sqrt{m}$ and $((u-(1 /(l m-(1)$ l))))/v) $\sqrt{m}$ are

$$
\begin{array}{r}
((u+(1 /(k m-1 /(k-(1 / k m))))) / v) \sqrt{m}, \\
((u-(1 / l m-(1 /(l-(1 / l m))))) / v) \sqrt{m},
\end{array}
$$

respectively. No nearest vertex exists.
Proof. (i) For the right side of $(u / v) \sqrt{m}$, we assume that there exists an edge $(u / v) \sqrt{m} \longrightarrow(x / y) \sqrt{m}$ in $G(\infty$, $(u / v) \sqrt{m})$ and $(u / v) \sqrt{m}<(x / y) \sqrt{m}$. We can write $(x / y)$ $\sqrt{m}$ in the form

$$
\frac{x}{y} \sqrt{m}=\frac{u}{v} \sqrt{m}+\frac{v x}{v y} \sqrt{m}-\frac{u y}{v y} \sqrt{m}=\frac{u+((v x-u y) / y)}{v} \sqrt{m}
$$

With this and the fact that $u y<v x$, we can replace $(x / y) \sqrt{m}$ with

$$
\frac{u+(t / s)}{v} \sqrt{m}=\frac{s u+t}{s v} \sqrt{m},
$$

where $t / s$ is in $\mathbb{Q}^{+}$. Let $d$ be the greatest common divisor of $s u+t$ and $s v$; then, we get $(s u+t / d, s v / d)=1$ and

$$
\frac{u}{v} \sqrt{m} \longrightarrow \frac{(s u+t) / d}{s v / d} \sqrt{m} \text {. }
$$

Theorem 1 gives the conditions when this edge exists. Since $(m, v)=1$, we have $m+v$ so case (i) in Theorem 1 cannot happen. Then, we thus consider case (ii). In this case, we have $(s u+t) / d \equiv \pm m u^{2} \bmod v$ and $u(s v / d)-v((s u+$ $t) / d)= \pm v$.

If $(s u+t / d) \equiv m u^{2} \bmod v$ and $u(s v / d)-v((s u+t) / d)=$ $v$, then $t=-d$ which implies $(s u-d) / d \equiv m u^{2} \bmod v$. As $m u^{2}+k m u+1 \equiv 0 \bmod v$, we have $(s u-d) / d \equiv-k m u-1$ $\bmod v$, that is, $s u \equiv-d k m u \bmod v$. Since $(u, v)=1$, we get $s \equiv-d k m \bmod v$. In other words, $s=-d k m-v z$ for some integer $z$. Thus,

$$
\frac{t}{s}=\frac{-d}{-d k m-v z}=\frac{1}{k m+(v z / d)} .
$$

We will find the largest value of $t / s$ by defining a function $f: \mathbb{R}^{+} \cup\{0\} \longrightarrow \mathbb{R}$

$$
f(z)=\frac{u+(1 /(k m+(v z / d)))}{v} \sqrt{m}
$$

The derivative of $f$ is $f^{\prime}(z)=\left(-d /(d k m+v z)^{2}\right) \sqrt{m}$, which is negative for every nonnegative $z$. This implies that the maximum occurs at $z=0$ and maximum value is

$$
\frac{u+(1 / k m)}{v} \sqrt{m}=\frac{k m u+1}{k m v} \sqrt{m}
$$

By Theorem 1, it now suffices to show that $(k m u+1) / k m v$ is an irreducible fraction. Thus,

$$
\frac{u+(1 / k m)}{v} \sqrt{m}
$$

is a vertex in $G(\infty,(u / v) \sqrt{m})$ and is the farthest one joined with $(u / v) \sqrt{m}$. We also see that

$$
\lim _{z \longrightarrow \infty} \frac{u+(1 /(k m+(v z / v z)))}{v} \sqrt{m}=\frac{u}{v} \sqrt{m} .
$$

This implies that there is no such nearest point joined with the vertex $(u / v) \sqrt{m}$.

If $(s u+t) / d \equiv-m u^{2} \bmod v$ and $u(s v / d)-v((s u+t) /$ $d)=-v$, then $t=d$, which implies $(s u+d) / d \equiv-m u^{2}$ $\bmod v$. We have $m u^{2}+k m u+1 \equiv 0 \bmod v$. This implies that $(s u+d) / d \equiv k m u+1 \bmod v$; that is, $s u \equiv d k m u \bmod v$. The 
fact that $(u, v)=1$ implies that $s \equiv d k m \bmod v$. Therefore, $s=d k m+v z$ for some $z$ in $\mathbb{N} \cup\{0\}$. Hence,

$$
\frac{t}{s}=\frac{d}{d k m+v z}=\frac{1}{k m+(v z / d)} \text {. }
$$

The proof is similar to the previous case. Next, we will consider the left side of $(u / v) \sqrt{m}$. Assume that there exists an edge

$$
\frac{u}{v} \sqrt{m} \longrightarrow \frac{x}{y} \sqrt{m}
$$

in $G(\infty,(u / v) \sqrt{m})$ and $(u / v) \sqrt{m}>(x / y) \sqrt{m}$. We can replace $(x / y) \sqrt{m}$ with

$$
\frac{u-(p / q)}{v} \sqrt{m}=\frac{q u-p}{q v} \sqrt{m},
$$

where $p / q$ is in $\mathbb{Q}^{+}$. Let $c$ be the greatest common divisor of $q u-p$ and $q v$; then, we get $((q u-p) / c, q v / c)=1$ and

$$
\frac{u}{v} \sqrt{m} \longrightarrow \frac{(q u-p) / c}{q v / c} \sqrt{m}
$$

By Theorem 1, case (i) cannot happen. So, we will consider case $(i i)$. Then, we have $(q u-p) / c \equiv \pm m u^{2} \bmod v$ and $u(q v / c)-v((q u-p) / c)= \pm v$.

If $(q u-p) / c \equiv m u^{2} \bmod v$ and $u(q v / c)-v((q u-p) / c)$ $=v$, then $p=c$ which implies $(q u-c) / c \equiv m u^{2} \bmod v$. As $m u^{2}-l m u+1 \equiv 0 \bmod v$, we have $(q u-c) / c \equiv \operatorname{lm} u-1$ $\bmod v$; that is, $q u \equiv c l m u \bmod v$. Since $(u, v)=1$, we get $q \equiv c l m \bmod v$. In other words, $q=c l m+v z$ for some integer $z$. Thus,

$$
\frac{p}{q}=\frac{c}{c l m+v z}=\frac{1}{l m+(v z / c)}
$$

We define a function $f: \mathbb{R}^{+} \cup\{0\} \longrightarrow \mathbb{R}$,

$$
f(z)=\frac{u-(1 /(\operatorname{lm}+(v z / c)))}{v} \sqrt{m} .
$$

The derivative of $f$ is $f^{\prime}(z)=\left(c /(c l m+v z)^{2}\right) \sqrt{m}$, which is positive for every nonnegative $z$. This implies that the minimum occurs at $z=0$ and minimum value is

$$
\frac{u-(1 / l m)}{v} \sqrt{m}=\frac{\operatorname{lm} u-1}{\operatorname{lm} v} \sqrt{m} .
$$

By Theorem 1, it now suffices to show that $(\operatorname{lmu}-1) / l m v$ is an irreducible fraction. Thus,

$$
\frac{u-(1 / l m)}{v} \sqrt{m}
$$

is a vertex in $G(\infty,(u / v) \sqrt{m})$ and is the farthest one joined with $(u / v) \sqrt{m}$. We also see that

$$
\lim _{z \longrightarrow \infty} \frac{u-(1 /(\operatorname{lm}+(v z / c)))}{v} \sqrt{m}=\frac{u}{v} \sqrt{m} .
$$

This implies that there is no such nearest point joined with the vertex $(u / v) \sqrt{m}$.

If $(q u-p) / c \equiv-m u^{2} \bmod v$ and $u(q v / c)-v((q u-p) /$ $c)=-v$, then $p=-c$ which implies $(q u+c) / c \equiv-m u^{2}$ $\bmod v$. We have $m u^{2}-l m u+1 \equiv 0 \bmod v$. This implies that $(q u+c) / c \equiv-\operatorname{lm} u+1 \bmod v$; that is, $q u \equiv-c l m u \bmod v$.
The fact that $(u, v)=1$ implies that $q \equiv-c l m \bmod v$. Therefore, $q=-c l m-v z$ for some $z$ in $\mathbb{N} \cup\{0\}$. Hence,

$$
\frac{p}{q}=\frac{-c}{-c l m-v z}=\frac{1}{l m+(v z / c)} \text {. }
$$

This case is done by using a similar argument to that of the previous case.

Corollary 3. Let $u$ and $v$ be relatively prime and $m$ prime such that $(m, v)=1$. If there are integers $k, l$ such that $m u^{2}+$ $k m u+1 \equiv 0 \bmod v$ and $m u^{2}-l m u+1 \equiv 0 \bmod v$, then

$$
\begin{aligned}
& \varphi(z)=\frac{-u \sqrt{m} z+\left(\left(m u^{2}+k m u+1\right) / v\right)}{-v z+(u+k) \sqrt{m}}, \\
& \psi(z)=\frac{-u \sqrt{m} z+\left(\left(m u^{2}-l m u+1\right) / v\right)}{-v z+(u-l) \sqrt{m}},
\end{aligned}
$$

are elements of $H(\sqrt{m})$. Moreover, $\varphi^{i+1}((u / v) \sqrt{m})$, $\psi^{i+1}((u / v) \sqrt{m})$ are the farthest vertices which can be joined with $\varphi^{i}((u / v) \sqrt{m})$ and $\psi^{i}((u / v) \sqrt{m})$, respectively, where $i \in \mathbb{N}$.

Corollary 4. Let $u$ and $v$ be relatively prime and $m$ prime. If $m u^{2}+k m u+1 \equiv 0 \bmod v$ where $1<k \leq v$, then there is an infinite path of minimal length:

$$
\begin{aligned}
\frac{1}{0} \sqrt{m} & \longrightarrow \frac{u}{v} \sqrt{m} \longrightarrow \frac{u+(1 / k m)}{v} \sqrt{m} \\
& \longrightarrow \frac{u+((k m-(1 / k)) / 1)}{v} \sqrt{m} \\
& \longrightarrow \frac{u+(1 /(k m-(1 /(k-(1 / k m)))))}{v} \sqrt{m} \longrightarrow \cdots
\end{aligned}
$$

whose vertices are in the set

$$
\begin{aligned}
V_{1}:= & \bigcup_{n=1}^{\infty}\left\{\frac{u+T_{n}(0)}{v} \sqrt{m}: T_{n}=t_{0} t_{1} \ldots t_{n}, t_{0}(z)=z, t_{i}(z)\right. \\
= & \left.\frac{-1}{-k m+z m} ; i \in\{1,2, \cdots, n\}\right\} \\
& \bigcup\left\{\infty, \frac{u}{v} \sqrt{m}\right\} \text { in } G\left(\infty, \frac{u}{v} \sqrt{m}\right) .
\end{aligned}
$$

Corollary 5. Let $u$ and $v$ be relatively prime and $m$ prime. If $m u^{2}-l m u+1 \equiv 0 \bmod v$ where $1<l \leq v$, then there is an infinite path of minimal length:

$$
\begin{aligned}
\ldots & \longleftarrow \frac{u-(1 /(\operatorname{lm}-(1 /(l-(1 / l m)))))}{v} \sqrt{m} \\
& \longleftarrow \frac{u-(1 /(\operatorname{lm}-(1 / l)))}{v} \sqrt{m} \longleftarrow \frac{u-(1 / l m)}{v} \sqrt{m} \\
& \longleftarrow \frac{u}{v} \sqrt{m} \longleftarrow \frac{1}{0} \sqrt{m},
\end{aligned}
$$


whose vertices are in the set

$$
\begin{aligned}
V_{2}:= & \bigcup_{n=1}^{\infty}\left\{\frac{u-T_{n}(0)}{v} \sqrt{m}: T_{n}=t_{0} t_{1} \ldots t_{n}, t_{0}(z)=z, t_{i}(z)\right. \\
= & \left.\frac{-1}{-l m+z m} ; i \in\{1,2, \ldots, n\}\right\} \\
& \bigcup\left\{\infty, \frac{u}{v} \sqrt{m}\right\} \text { in } G\left(\infty, \frac{u}{v} \sqrt{m}\right) .
\end{aligned}
$$

\section{Infinite Path of Minimal Length in $G(\infty,(u / v) \sqrt{m})$ Where $(m, v)=m$}

In the previous section, we provided the existence of infinite path of minimal length in the suborbital graph $G(\infty,(u / v) \sqrt{m})$ where $(m, v)=1$. We find that the existence property is also valid for the suborbital graph $G(\infty,(u / v) \sqrt{m})$ where $(m, v)=m$ in very close analogy.

Theorem 6. Let $u$ and $v$ be relatively prime and $m$ prime such that $(m, v)=m$, and let $k, l$ be the integers uniquely determined in Theorem 8 . Then we have the following results in $G(\infty,(u / v) \sqrt{m})$ :

(i) The farthest vertices which can be joined with $(u / v) \sqrt{m}$ on the right and the left are

$$
\begin{aligned}
& \frac{u+(1 / k)}{v} \sqrt{m}, \\
& \frac{u-(1 / l)}{v} \sqrt{m},
\end{aligned}
$$

respectively. No nearest vertex exists.

(ii) The farthest vertices which can be joined with $((u+$ $(1 / k)) / v) \sqrt{m}$ and $((u-(1 / l)) / v) \sqrt{m}$ are

$$
\begin{gathered}
\frac{u+(1 /(k-(1 / k)))}{v} \sqrt{m}, \\
\frac{u-(1 /(l-(1 / l)))}{v} \sqrt{m},
\end{gathered}
$$

respectively. No nearest vertex exists.

(iii) The farthest vertices which can be joined with $((u+$ $(1 /(k-(1 / k)))) / v) \sqrt{m}$ and $((u-(1 /(l-(1 / l)))) / v)$ $\sqrt{m}$ are

$$
\begin{gathered}
\frac{u+(1 /(k-(1 /(k-(1 / k)))))}{v} \sqrt{m}, \\
\frac{u-(1 /(l-(1 /(l-(1 / l)))))}{v} \sqrt{m},
\end{gathered}
$$

respectively. No nearest vertex exists.
Corollary 6. Let $u$ and $v$ be relatively prime and $m$ prime such that $(m, v)=m$. If there are integers $k, l$ such that $u^{2}+$ $k u+1 \equiv 0 \bmod v$ and $u^{2}-l u+1 \equiv 0 \bmod v$, then

$$
\begin{aligned}
& \lambda(z)=\frac{-u z+\left(\left(u^{2}+k u+1\right) / v\right) \sqrt{m}}{\sqrt{m} z+(u+k)}, \\
& \gamma(z)=\frac{-u z+\left(\left(u^{2}-l u+1\right) / v\right) \sqrt{m}}{-(v / m) \sqrt{m} z+(u-l)},
\end{aligned}
$$

are elements of $H(\sqrt{m})$. Moreover, $\lambda^{i+1}((u / v) \sqrt{m})$, $\gamma^{i+1}((u / v) \sqrt{m})$ are the farthest vertices which can be joined with $\lambda^{i}((u / v) \sqrt{m})$ and $\gamma^{i}((u / v) \sqrt{m})$, respectively, where. $i=1,2, \ldots$.

Corollary 7. Let $u$ and $v$ be relatively prime and $m$ prime. If $u^{2}+k u+1 \equiv 0 \bmod v$ where $1<k \leq v$, then there is an infinite path of minimal length:

$$
\begin{gathered}
\frac{1}{0} \sqrt{m} \longrightarrow \frac{u}{v} \sqrt{m} \longrightarrow \frac{u+()}{v} \sqrt{m} \longrightarrow \frac{u+(1 /(k-(1 / k)))}{v} \sqrt{m} \\
\longrightarrow \frac{u+(1 /(k-(1 /(k-(1 / k)))))}{v} \sqrt{m} \longrightarrow \cdots,
\end{gathered}
$$

whose vertices are in the set

$$
\begin{aligned}
W_{1}:= & \bigcup_{n=1}^{\infty}\left\{\frac{u+T_{n}(0)}{v} \sqrt{m}: T_{n}=t_{0} t_{1} \ldots t_{n}, t_{0}(z)=z, t_{i}(z)\right. \\
= & \left.\frac{-1}{-k+z} ; i \in\{1,2, \ldots, n\}\right\} \\
& \bigcup\left\{\infty, \frac{u}{v} \sqrt{m}\right\} \text { in } G\left(\infty, \frac{u}{v} \sqrt{m}\right) .
\end{aligned}
$$

Corollary 8. Let $u$ and $v$ be relatively prime and $m$ prime. If $u^{2}-l u+1 \equiv 0 \bmod v$ where $1<l \leq v$, then there is an infinite path of minimal length:

$$
\begin{aligned}
& \cdots \longleftarrow \frac{u-(1 /(l-(1 /(l-(1 / l)))))}{v} \sqrt{m} \\
& . \longleftarrow \frac{u-(1 /(l-(1 / l)))}{v} \sqrt{m} \longleftarrow \frac{u-(1 / l)}{v} \sqrt{m} \\
& \longleftarrow \frac{u}{v} \sqrt{m} \longleftarrow \frac{1}{0} \sqrt{m},
\end{aligned}
$$

whose vertices are in the set

$$
\begin{aligned}
W_{2} & :=\bigcup_{n=1}^{\infty}\left\{\frac{u-T_{n}(0)}{v} \sqrt{m}: T_{n}=t_{0} t_{1} \ldots t_{n}, t_{0}(z)=z, t_{i}(z)\right. \\
& \left.=\frac{-1}{-l+z} ; i \in\{1,2, \cdots, n\} \cup\left\{\infty \frac{u}{v} \sqrt{m}\right\}\right\} \text { in } G\left(\infty, \frac{u}{v} \sqrt{m}\right) .
\end{aligned}
$$




\section{Continued Fractions and Recurrence Relations}

From results in Sections 3 and 4, we have that any vertex on the infinite path of minimal length can be represented by a continued fraction expansion. As a continued fraction is related to recurrence relations, we use them to investigate vertices on the infinite path of minimal length. We conclude this section by finding the limit point of the sequence of the vertices.

Let $\left\{a_{n}\right\}_{n \in \mathbb{N}^{N}}\left\{b_{n}\right\}_{n \in \mathbb{N}_{0}}$ be sequences of complex numbers with $a_{n} \neq 0$ for $n \geq 1$ and $\left\{t_{m}\right\}_{n \in \mathbb{N}_{0}},\left\{T_{m}\right\}_{n \in \mathbb{N}_{0}}$ be sequences of Möbius transformations defined as follows:

$$
\begin{aligned}
& t_{0}(z)=b_{0}+z, \\
& t_{n}(z)=\frac{a_{n}}{b_{n}+z}, \quad \text { for } n \in \mathbb{N}, \\
& T_{0}(z)=t_{0}(z), \\
& T_{n}(z)=T_{n-1}\left(t_{n}(z)\right), \quad \text { for } n \in \mathbb{N} .
\end{aligned}
$$

We consider $T_{0}(0), T_{1}(0), T_{2}(0), T_{3}(0)$ and so on and form a continued fraction of the form

$$
T_{n}(0)=b_{0}+\frac{a_{1}}{b_{1}+\frac{a_{2}}{b_{2}+\frac{a_{3}}{b_{3}+\frac{a_{4}}{\ddots+\frac{a_{n}}{b_{n}}}}}}
$$

For convenience, we denote this by

$$
b_{0}+\frac{a_{1}}{b_{1}}+\frac{a_{2}}{b_{2}}+\frac{a_{3}}{b_{3}}+\cdots+\frac{a_{n}}{b_{n}} .
$$

In [10], the $n^{\text {th }}$ numerator $A_{n}$ and the $n^{\text {th }}$ denominator $B_{n}$ of a continued fraction as in (43) are defined by the recurrence relations

$$
\left[\begin{array}{l}
A_{n} \\
B_{n}
\end{array}\right]=b_{n}\left[\begin{array}{l}
A_{n-1} \\
B_{n-1}
\end{array}\right]+a_{n}\left[\begin{array}{c}
A_{n-2} \\
B_{n-2}
\end{array}\right], \quad n=1,2,3, \ldots,
$$

with initial conditions

$$
\begin{aligned}
A_{-1} & =1, \\
B_{-1} & =0, \\
A_{0} & =b_{0}, \\
B_{0} & =1 .
\end{aligned}
$$

For a given sequence $\left\{z_{n}\right\}_{n \in \mathbb{N}_{0}}, T_{n}\left(z_{n}\right)$ can be written as

$$
T_{n}\left(z_{n}\right)=\frac{A_{n}+A_{n-1} z_{n}}{B_{n}+B_{n-1} z_{n}}, \quad n=0,1,2, \ldots,
$$

and then

$$
T_{n}(0)=\frac{A_{n}}{B_{n}}
$$

Now we consider infinite paths in suborbital graph $G(\infty,(u / v) \sqrt{m})$. For the case when $(m, v)=1$, the infinite path of minimal length for the right direction in Corollary 4 gives $a_{n}=-(1 / m)$ and $b_{n}=-k$ for $n \geq 1$. By recurrence relations in (45), we obtain $B_{n}=-m A_{n+1}$, and then, we have a vertex on this path:

$$
\frac{u+T_{n}(0)}{v} \sqrt{m}=\frac{A_{n+1} m u-A_{n}}{A_{n+1} m v} \sqrt{m} .
$$

Similarly, we have a vertex on the infinite path of minimal length for the left direction is

$$
\frac{u-T_{n}(0)}{v} \sqrt{m}=\frac{A_{n+1} l u+A_{n}}{A_{n+1} l v} \sqrt{m} .
$$

Theorem 7. If $(m, v)=1$ and $k \geq 2$, then we have

$$
\begin{aligned}
A_{n}= & \left(-\frac{1}{m}\right)^{n} 2^{1-n} \sum_{t=1}^{n}\left(k m+\sqrt{(k m)^{2}-4 m}\right)^{n-t} \\
& \cdot\left(k m-\sqrt{(k m)^{2}-4 m}\right)^{t-1} .
\end{aligned}
$$

Proof. From the recurrence relation, we have

$$
m A_{n+2}+k m A_{n+1}+A n=0
$$

with $A_{-1}=1$ and $A_{0}=0$. The characteristic equation for the relation (53) is

$$
m x^{2}+k m x+1=0
$$

which gives two roots

$$
\begin{aligned}
& x_{1}=\frac{-k m+\sqrt{(k m)^{2}-4 m}}{2 m}, \\
& x_{2}=\frac{-k m-\sqrt{(k m)^{2}-4 m}}{2 m} .
\end{aligned}
$$

Then, any solution of (9) have the form

$$
\begin{aligned}
A_{n}= & \alpha\left(\frac{-k m+\sqrt{(k m)^{2}-4 m}}{2 m}\right)^{n} \\
& +\beta\left(\frac{-k m-\sqrt{(k m)^{2}-4 m}}{2 m}\right)^{n} .
\end{aligned}
$$

By using the initial conditions, we have

$$
\begin{aligned}
A_{0}= & \alpha+\beta=0, \\
A_{1}= & \alpha\left(-k m+\sqrt{(k m)^{2}-4 m}\right) \\
& +\beta\left(-k m-\sqrt{(k m)^{2}-4 m}\right)=-2,
\end{aligned}
$$

which implies

$$
\begin{aligned}
& \alpha=\frac{-1}{\sqrt{(k m)^{2}-4 m}}, \\
& \beta=\frac{1}{\sqrt{(k m)^{2}-4 m}} .
\end{aligned}
$$


Hence, we get

$$
\begin{aligned}
A_{n}= & \left(\frac{-1}{2 m}\right)^{n} \frac{1}{\sqrt{(k m)^{2}-4 m}}\left[\left(k m+\sqrt{(k m)^{2}-4 m}\right)^{n}\right. \\
& \left.-\left(k m-\sqrt{(k m)^{2}-4 m}\right)^{n}\right] .
\end{aligned}
$$

Since $\left(k m+\sqrt{(k m)^{2}-4 m}\right)^{n}-\left(k m-\sqrt{(k m)^{2}-4 m}\right)^{n}$ is equal to

$$
\begin{gathered}
2 \sqrt{(k m)^{2}-4 m} \sum_{t=1}^{n}\left(k m+\sqrt{(k m)^{2}-4 m}\right)^{n-t} \\
\cdot\left(k m-\sqrt{(k m)^{2}-4 m}\right)^{t-1},
\end{gathered}
$$

then we obtain

$$
\begin{aligned}
A_{n}= & \left(-\frac{1}{m}\right)^{n} 2^{1-n} \sum_{t=1}^{n}\left(k m+\sqrt{(k m)^{2}-4 m}\right)^{n-t} \\
& \cdot\left(k m-\sqrt{(k m)^{2}-4 m}\right)^{t-1} .
\end{aligned}
$$

Theorem 8. If $(m, v)=1$ and $l \geq 2$, then we have

$$
\begin{aligned}
A_{n}= & \left(-\frac{1}{m}\right)^{n} 2^{1-n} \sum_{t=1}^{n}\left(\operatorname{lm}+\sqrt{(\operatorname{lm})^{2}-4 m}\right)^{n-t} \\
& \cdot\left(l m-\sqrt{(l m)^{2}-4 m}\right)^{t-1} .
\end{aligned}
$$

Next, we consider case $(m, v)=m$. By Corollary 7 , we have $a_{n}=-1, b_{n}=-k$ for $n \geq 1$. So we get $B_{n}=A_{n+1}$ from recurrence relations in (45). Then, a vertex on the infinite path of minimal length for the right direction is

$$
\frac{u+T_{n}(0)}{v} \sqrt{m}=\frac{A_{n+1} u-A_{n}}{A_{n+1} v} \sqrt{m} .
$$

Likewise, a vertex on the infinite path of minimal length for the left direction is

$$
\frac{u-T_{n}(0)}{v} \sqrt{m}=\frac{A_{n+1} u+A_{n}}{A_{n+1} v} \sqrt{m} .
$$

Theorem 9. If $(m, v)=m$ and $k \geq 2$, then we have

$$
A_{n}=\left\{\begin{array}{l}
(-1)^{n} n, k=2 \\
(-1)^{n} 2^{1-n} \sum_{t=1}^{n}\left(k+\sqrt{(k)^{2}-4}\right)^{n-t}\left(k-\sqrt{(k)^{2}-4}\right), \quad k>2 .
\end{array}\right.
$$

Theorem 10. If $(m, v)=m$ and $l \geq 2$, then we have

$$
A_{n}=\left\{\begin{array}{l}
(-1)^{n} n, l=2, \\
(-1)^{n} 2^{1-n} \sum_{t=1}^{n}\left(l+\sqrt{(l)^{2}-4}\right)^{n-t}\left(l-\sqrt{(l)^{2}-4}\right)^{t-1}, \quad l>2 .
\end{array}\right.
$$

Having characterized the vertices on the infinite path of minimal length, we investigate the limit point of this path by using the Śleszyński-Pringsheim theorem.

Theorem 11 [10] (Śleszyński-Pringsheim). The continued fraction

$$
\frac{a_{1}}{b_{1}}+\frac{a_{2}}{b_{2}}+\frac{a_{3}}{b_{3}}+\cdots+\frac{a_{n}}{b_{n}}+\cdots
$$

converges to some valued $f$ with $|f| \leq 1$ if

$$
\left|b_{n}\right| \geq 1+\left|a_{n}\right|, \quad n \geq 1 \text {. }
$$

Corollary 12. The sequence of the vertices of infinite path of minimal length (30) converges to

$$
\frac{2 m u+m k-\sqrt{m k^{2}-4} \cdot \sqrt{m}}{2 m v} \sqrt{m} \text {. }
$$

Proof. Since we have $a_{n}=-1 / m$ and $b_{n}=-k$ where $m$ is prime and $k \geq 2$, we get $\left|b_{n}\right| \geq 1+\left|a_{n}\right|$ for all $n \geq 1$. By Theorem 11 , the continued fraction

$$
\frac{1}{k m}-\frac{1}{k}-\frac{1}{k m}-\cdots
$$

converges to $f$ with $|f| \leq 1$; that is, $\lim _{n \longrightarrow \infty} T_{n}(0)=f$. As we know

$$
T_{n}(0)=\frac{1}{k m-m T_{n-1}(0)},
$$

$T_{n}(0)\left(k m-m T_{n-1}(0)\right)=1$, and since $\lim _{n \rightarrow \infty} T_{n}(0)=$ $\lim _{n \rightarrow \infty} T_{n-1}(0)$, we have $f(k m-m f)=1$. Hence, $m f^{2}-$ $m k f+1=0$ and

$$
f=\frac{m k \pm \sqrt{m^{2} k^{2}-4 m}}{2 m} .
$$


As $k \geq 2$ and $|f| \leq 1$, we get $f=\left(m k-\sqrt{m^{2} k^{2}-4 m}\right) / 2 m$. Therefore, we obtain that the sequence of the vertices of infinite path of minimal length (30) converges to

$$
\begin{aligned}
& \frac{u+\left(\left(m k-\sqrt{m^{2} k^{2}-4 m}\right) / 2 m\right)}{v} \sqrt{m} \\
& =\frac{2 m u+m k-\sqrt{m k^{2}-4} \cdot \sqrt{m}}{2 m v} \sqrt{m} .
\end{aligned}
$$

Corollary 13. The sequence of the vertices of infinite path of minimal length (32) converges to

$$
\frac{2 m u-m l+\sqrt{m l^{2}-4} \cdot \sqrt{m}}{2 m v} \sqrt{m} \text {. }
$$

Corollary 14. The sequence of the vertices of infinite path of minimal length (38) converges to

$$
\frac{2 u+k-\sqrt{k^{2}-4}}{2 v} \sqrt{m} \text {. }
$$

Corollary 15. The sequence of the vertices of infinite path of minimal length (40) converges to

$$
\frac{2 u-l+\sqrt{l^{2}-4}}{2 v} \sqrt{m} \text {. }
$$

We observe that the limit points in Corollaries 23 and 24 are not in the set $\sqrt{m} \widehat{\mathbb{Q}}$, but the limit points in Corollaries 25 and 26 will be in the set 2 if $k=l=2$.

\section{Data Availability}

There are no data for supporting this study.

\section{Conflicts of Interest}

The authors declare that they have no conflicts of interest.

\section{Acknowledgments}

This research was supported by Chiang Mai University.

\section{References}

[1] E. Hecke, "Über die Bestimmung Dirichletscher Reihen durch ihre Funktionalgleichung," Mathematische Annalen, vol. 112, no. 1, pp. 664-699, 1936.

[2] L. A. Parson, "Normal congruence subgroups of the Hecke groups $G\left(2^{(1 / 2)}\right)$ and $G\left(3^{(1 / 2)}\right)$," Pacific Journal of Mathematics, vol. 70, no. 2, pp. 481-487, 1977.

[3] D. Rosen, "A class of continued fractions associated with certain properly discontinuous groups," Duke Mathematical Journal, vol. 21, no. 3, pp. 549-563, 1954.

[4] R. Keskin, "On the parabolic class numbers of some Fuchsian groups," Note di Matematica, vol. 19, no. 2, pp. 275-283, 1999.

[5] G. A. Jones, D. Singerman, and K. Wicks, "The modular group and generalized farey graphs," in London Mathematical Society Lecture Note Series, vol. 160, pp. 316-338, 1991.
[6] C. C. Sims, "Graphs and finite permutation groups," Mathematische Zeitschrift, vol. 95, no. 1, pp. 76-86, 1967.

[7] M. Akbas, "On suborbital graphs for the modular group," Bulletin of the London Mathematical Society, vol. 33, no. 6, pp. 647-652, 2001.

[8] O. Yayenie, "Subgroups of some Fuchsian groups defined by two linear congruences," Conformal Geometry and Dynamics of the American Mathematical Society, vol. 11, no. 18, pp. 271-288, 2007.

[9] R. Keskin, "On suborbital graphs for some Hecke groups," Discrete Mathematics, vol. 234, no. 1-3, pp. 53-64, 2001.

[10] A. Cuyt, V. B. Petersen, B. Verdonk, H. Waadeland, and W. B. Jones, Handbook of Continued Fractions for Special Functions, Springer, New York, NY, USA, 2008. 


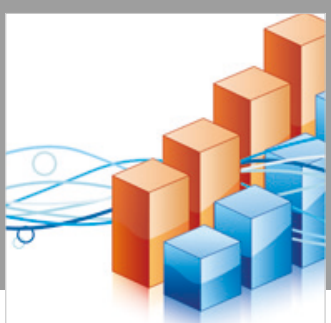

Advances in

Operations Research

\section{-n-m}
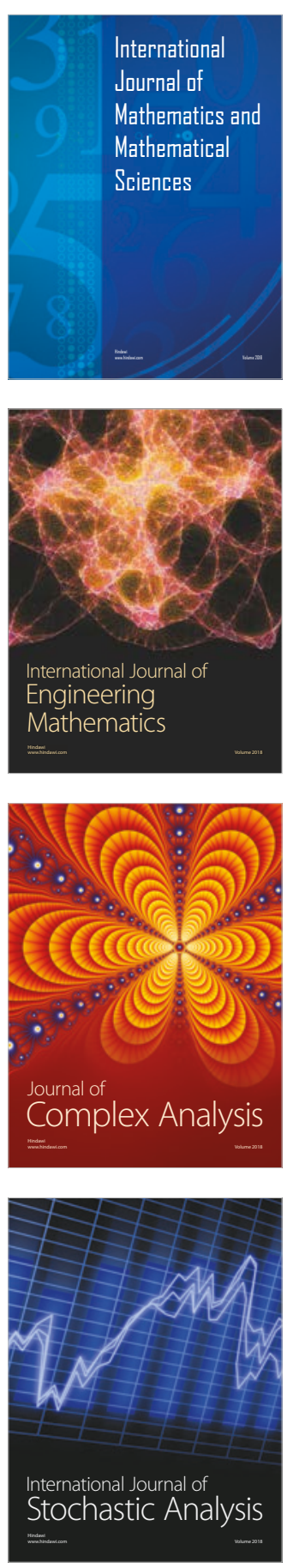
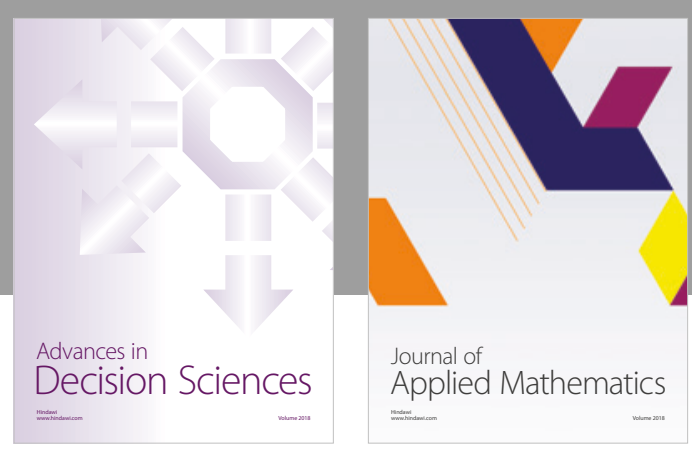

Journal of

Applied Mathematics
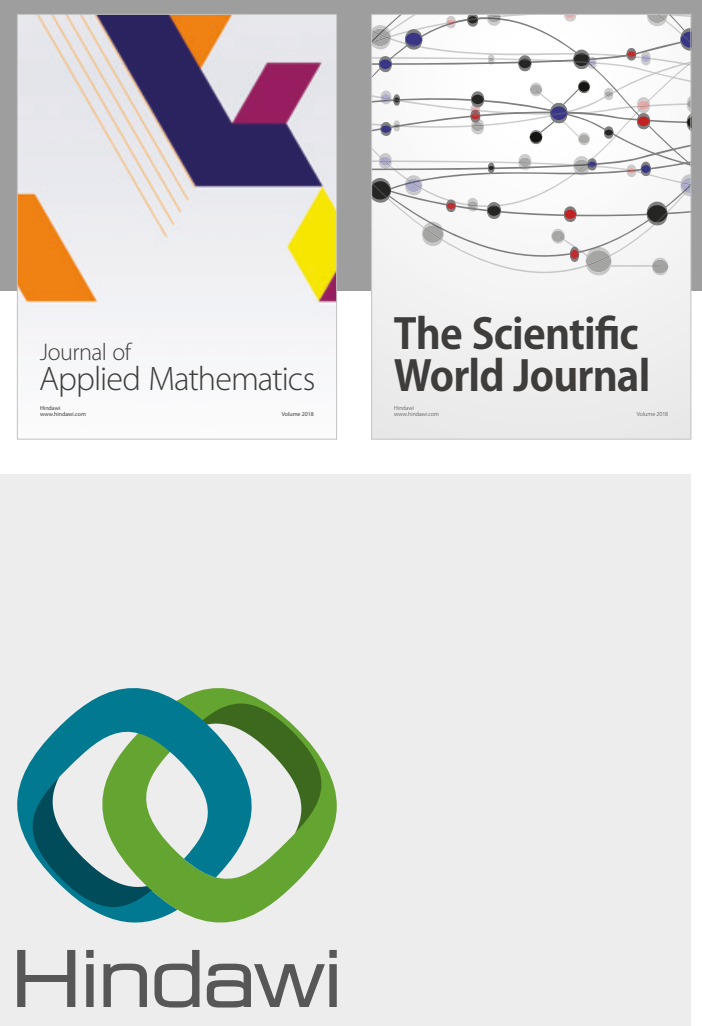

Submit your manuscripts at

www.hindawi.com

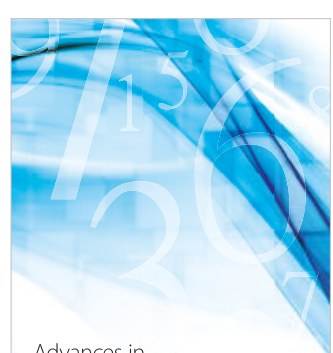

Advances in
Numerical Analysis
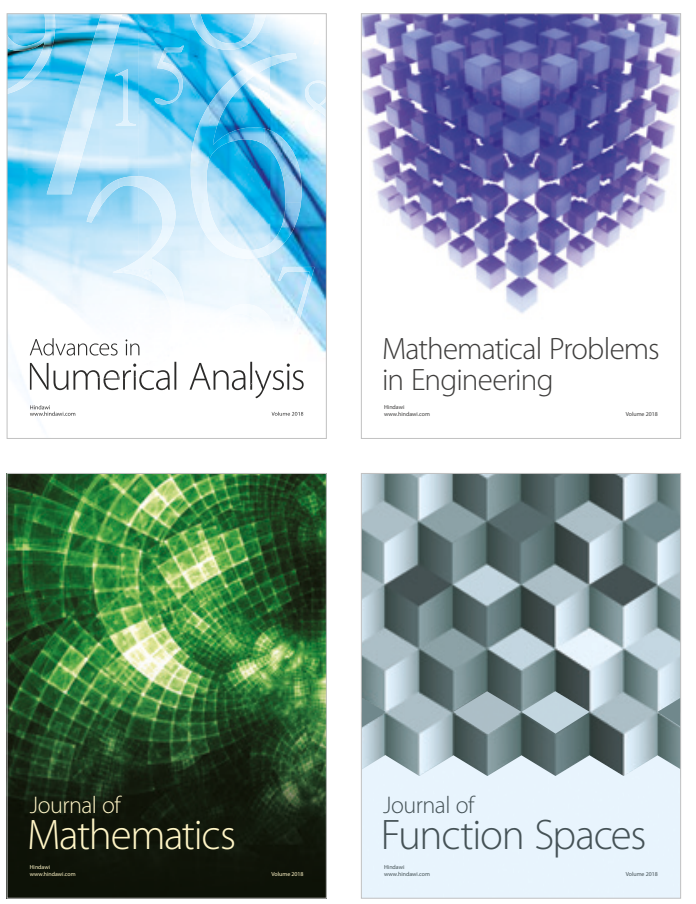

Mathematical Problems in Engineering

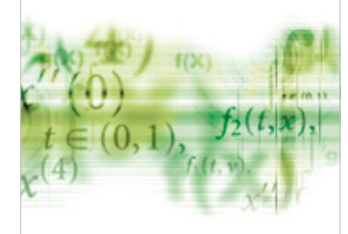

International Journal of

Differential Equations

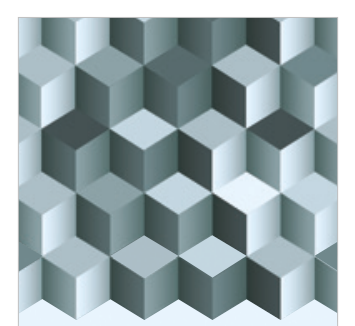

Journal of

Function Spaces

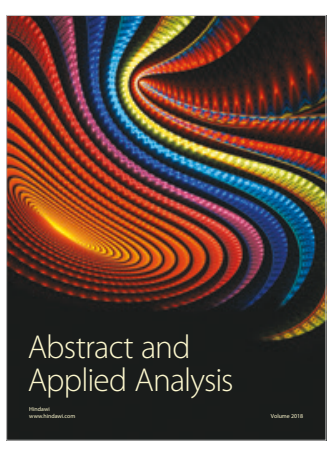

The Scientific

World Journal

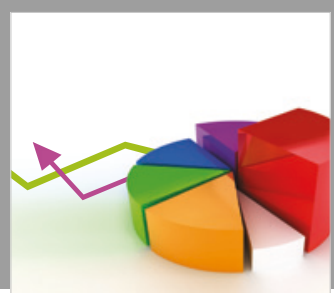

Journal of

Probability and Statistics
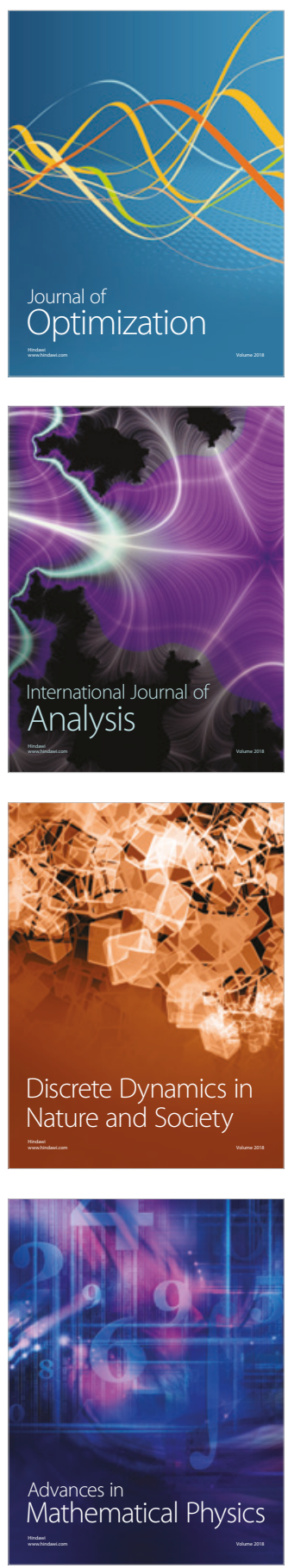